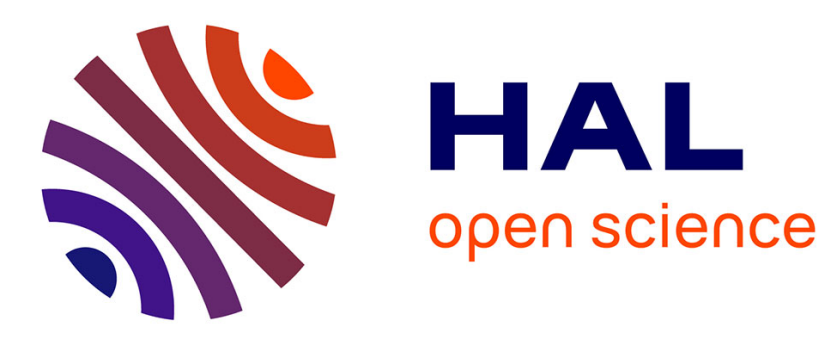

\title{
Extensional viscosity and thinning of a fiber suspension thread
}

\author{
Joris Château, Élisabeth Guazzelli, Henri Lhuissier
}

\section{To cite this version:}

Joris Château, Élisabeth Guazzelli, Henri Lhuissier. Extensional viscosity and thinning of a fiber suspension thread. Physical Review Fluids, 2021, 6 (4), 10.1103/PhysRevFluids.6.044307 . hal03366850

\section{HAL Id: hal-03366850 \\ https://hal.science/hal-03366850}

Submitted on 5 Oct 2021

HAL is a multi-disciplinary open access archive for the deposit and dissemination of scientific research documents, whether they are published or not. The documents may come from teaching and research institutions in France or abroad, or from public or private research centers.
L'archive ouverte pluridisciplinaire HAL, est destinée au dépôt et à la diffusion de documents scientifiques de niveau recherche, publiés ou non, émanant des établissements d'enseignement et de recherche français ou étrangers, des laboratoires publics ou privés. 


\title{
Extensional viscosity and thinning of a fiber suspension thread
}

\author{
Joris Château, ${ }^{1}$ Élisabeth Guazzelli, ${ }^{2}$ and Henri Lhuissier ${ }^{1, *}$ \\ ${ }^{1}$ Aix Marseille Univ, CNRS, IUSTI, Marseille, France \\ ${ }^{2}$ Université de Paris, CNRS, Matière et Systèmes Complexes (MSC) UMR 7057, Paris, France
}

(Dated: October 5, 2021)

\begin{abstract}
The extensional effective viscosity, $\eta_{\mathrm{e}}$, of a suspension of solid cylindrical fibers in a Newtonian liquid is measured from the gravitational stretching of a quasi-steady jet. We vary the lengthto-diameter aspect ratio of the fibers, $a$, from $\sim 10$ to $\sim 10^{2}$, and the particle volume fraction, $\phi$, in the range $a \phi \sim 0.1-2$. For low values of $\phi$, the extensional viscosity is found to agree with Batchelor's model for the dilute regime, which assumes non-interacting fibers aligned with the stretching direction, but for higher concentrations, $\eta_{\mathrm{e}}$ is found to increase much more strongly with increasing $\phi$ than predicted by available models assuming purely hydrodynamics interactions between the fibers. Additional experiments are performed on the breakup of an unstable capillary bridge for $a=11$. Although the variability in the bridge shape and the deviation from a viscous Newtonian dynamics in the late stage of the breakup increase significantly with increasing $\phi$, it is found that the mean total duration of the breakup is in good agreement with a Newtonian effective dynamics limited by the extensional viscosity, $\eta_{\mathrm{e}}$.
\end{abstract}

\section{INTRODUCTION}

The formation, deformation, and fragmentation of elongated structures, such as jets or threads, involve essentially extensional flows, for which the rheological response of the deformed medium may differ strongly from its response to a shear flow, in particular when the medium has a microstructure depending on the type of deformation. A typical example is that of a suspension of elongated particles, such as solid fibers, which, provided they are diluted enough, align with the straining direction in a purely extensional flow, while they tumble in a pure shear.

For fibers that are rigid, non-Brownian, suspended in a Newtonian liquid having a viscosity $\eta_{0}$, and sufficiently small to neglect inertial effects, the bulk response of the suspension is pseudo-Newtonian in all flows, in the sense that all the bulk effective stresses (except the undetermined bulk pressure) must be proportional to both $\eta_{0}$ and the actual deformation rate. Moreover, if the orientation of the particles has statistically the same symmetry as the bulk flow, the deviatoric part of the bulk stresses must have the same tensorial form as for a Newtonian liquid. Importantly, this condition is verified for a liquid thread containing fibers with an initially random orientation or for a jet extruded from a cylindrical nozzle that are both stretched parallel to their own axis, since the fibers are expected to evolve from the initial isotropic or partly aligned configuration to the final aligned state while preserving the axial symmetry of the flow. In this case,the extensional bulk rheology is fully defined by a single coefficient, the extensional effective viscosity $\eta_{\mathrm{e}} \equiv \tau_{i j} / 2 E_{i j}$ where $\boldsymbol{E}$ and $\boldsymbol{\tau}$ are the strain rate tensor and the deviatoric part of the viscous stress tensor, respectively. Note that from this definition, $\eta_{\mathrm{e}}$ is equal to the shear viscosity, $\eta_{\mathrm{s}}$, in the case of a Newtonian liquid.

In the absence of contact between the fibers, $\eta_{\mathrm{e}}$ can only result from hydrodynamic perturbations of the mean flow by the fibers, and the fibers can only interact via hydrodynamic forces. In the case of rigid particles, the effective viscosity depends only on the particle volume fraction, $\phi$, the length-to-diameter aspect ratio of the fibers, $a=l / d$, and the degree of alignement of the fibers. A series of expressions for $\eta_{\mathrm{e}}$ have been derived by Batchelor [2] and Shaqfeh \& Fredrickson [20] for different degrees of fiber dilution in a uni-axial straining flow $\left(\boldsymbol{E}=-\dot{\epsilon} / 2 \boldsymbol{e}_{\boldsymbol{x}} \boldsymbol{e}_{\boldsymbol{x}}-\dot{\epsilon} / 2 \boldsymbol{e}_{\boldsymbol{y}} \boldsymbol{e}_{\boldsymbol{y}}+\dot{\epsilon} \boldsymbol{e}_{\boldsymbol{z}} \boldsymbol{e}_{\boldsymbol{z}}\right)$. These authors have considered slender fibers $(a \gg 1)$, neglected inertia and frictional contact between the fibers, and assumed simplified hydrodynamic interactions between the fibers. Most models have also considered parallel fibers aligned with the stretching direction $\left(\boldsymbol{e}_{\boldsymbol{z}}\right)$. In the dilute limit $\left(a^{2} \phi / \ln a \ll 1\right)$, i. e. when the mean distance between neighboring fibers, $\sim d / \sqrt{\phi}$, is larger than the hydrodynamic range, $\sim l / \sqrt{\ln a}$, the interaction between the fibers can be neglected and the specific viscosity, $\eta_{\mathrm{e}} / \eta_{0}-1$, is proportional to $\phi$. By summing the additional stresses due to force-free, isolated fibers (i. e. stresslets, see [1, 5, 9]), Batchelor [2] obtained

$$
\frac{\eta_{\mathrm{e}}}{\eta_{0}}-1=\frac{2 \varepsilon Q(\varepsilon)}{9} a^{2} \phi, \quad \text { with } \quad \varepsilon=\frac{1}{\ln (2 a)},
$$

where the shape factor $Q(\varepsilon)=\frac{1+0.64 \varepsilon}{1-3 \varepsilon / 2}+1.659 \varepsilon^{2}+\mathcal{O}\left(\varepsilon^{3}\right)$ is of order one in practice. The alignement of the particles with the direction of the largest strain maximizes the dissipation. Due to the linearity of the stresses, equation (1) can be extended to any distribution of particle orientation. For isotropic particles, the specific viscosity is five times

\footnotetext{
* henri.lhuissier@univ-amu.fr
} 

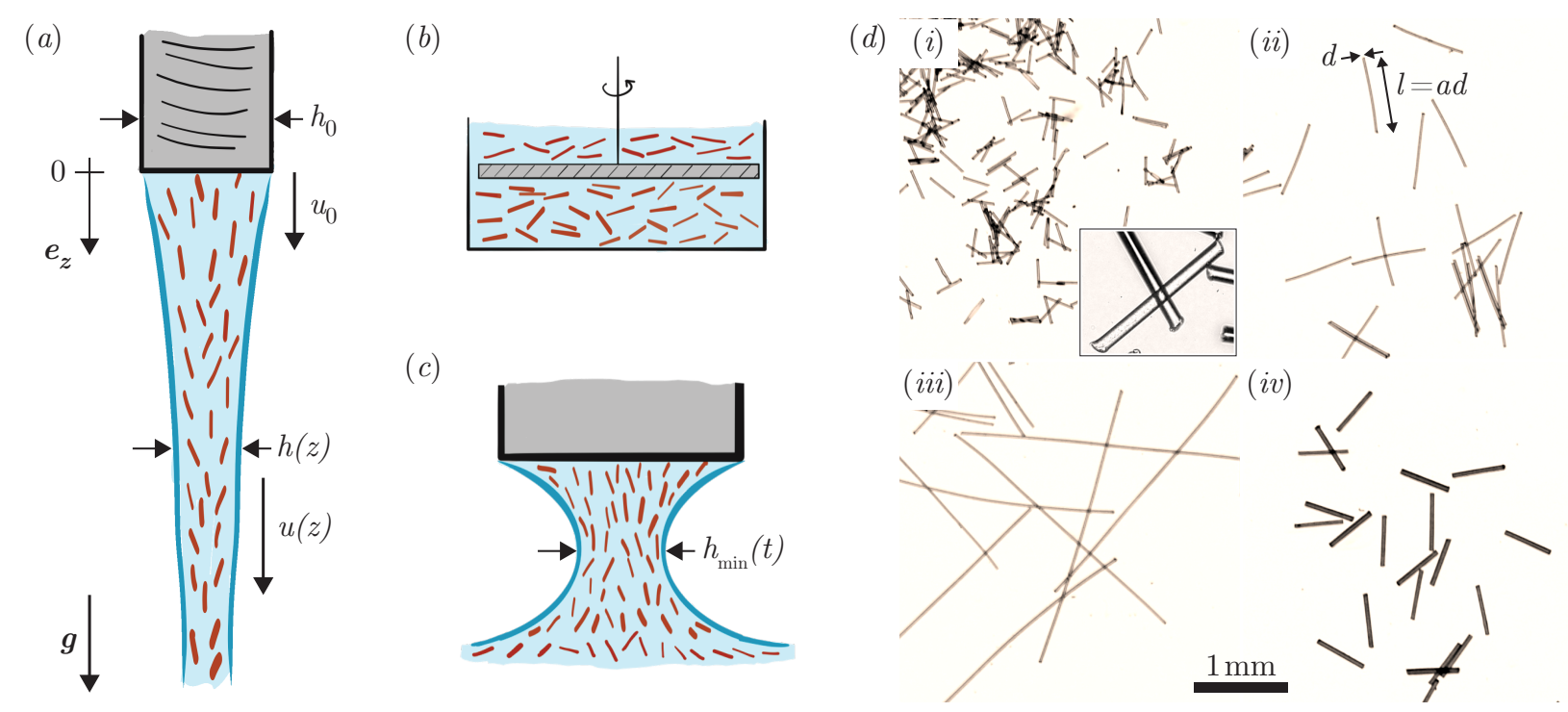

Figure 1. Setups and fibers. (a) Constant flow-rate jet stretched by gravity used for the extensional rheometry. (b) Shear cell used for the shear rheometry. (c) Capillary bridge breakup configuration. ( $d$ ) Cylindrical polyamide fibers used for the experiments: $(i) d=28.9 \mu \mathrm{m}, a=10.8$. (ii) $d=28.3 \mu \mathrm{m}, a=27.6$. (iii) $d=28.1 \mu \mathrm{m}, a=106$. (iv) $d=48.1 \mu \mathrm{m}, a=11.5$.

as small as that given by equation (1). Experiments with a jet stretched with a rotating cylinder [14, 23] or by gravity [17] have verified the validity of equation (1) for dilute suspensions of thin elongated fibers $(d \lesssim 10 \mu \mathrm{m}, a \sim 50-1200$, and $\phi \sim 5.10^{-4}-0.01$ ). Modifications of equation (1) have also been proposed to account for the weak hydrodynamic interactions between the fibers in the so-called semi-dilute regime where the mean distance between the fibers is smaller than the hydrodynamic range while remaining larger than the fibers diameter $(d \ll d / \sqrt{\phi} \lesssim l / \sqrt{\ln a})$. For semi-dilute suspensions of aligned fibers, Batchelor [2] and Shaqfeh \& Fredrickson [20] have obtained, respectively,

$$
\text { and } \quad \begin{aligned}
\frac{\eta_{\mathrm{e}}}{\eta_{0}}-1 & =\frac{4}{9} \frac{1}{\ln \pi / \phi} a^{2} \phi, \\
\frac{\eta_{\mathrm{e}}}{\eta_{0}}-1 & =\frac{4}{9} \frac{1}{\ln [\ln (1 / \phi) / \phi]+0.1585} a^{2} \phi,
\end{aligned}
$$

from a cell model approximation with a mean field cell size $d / \sqrt{\phi}$ and a multiple scattering model of the hydrodynamic interactions, respectively.

Beyond these purely hydrodynamic regimes, a different rheological behavior may be expected as $\phi$ is increased since solid contacts between the particles must become important at some point. Above solid fractions of order $1 / a[6,16,18]$, solid contacts may prevent the fibers from aligning, and even for a given orientation distribution of the particles, an increase in solid contacts between the particles is expected as $\phi$ becomes of order 1 or as $a$ is sufficiently increased. This should definitely affect the particle flow and hence the overall dissipation. This limit has been documented recently for a pure shear flow [21], but data are not available for straining flow.

These questions are precisely addressed in the present contribution by measuring the extensional effective viscosity of a suspension of solid cylindrical fibers in a Newtonian liquid from the gravitational stretching of a quasi-steady jet. These experiments are complemented by additional observations on the breakup of an unstable capillary bridge using the same fiber suspensions. The experimental methods are described in $\S I I$ and the results in $\S I I I$. Conclusions are drawn is $\S \mathrm{IV}$.

\section{MATERIALS AND EXPERIMENTAL METHODS}

We use cylindrical polyamide fibers manufactured for flocking applications (provided by the Pinfloc company). Their diameter, $d$, is varied between $\simeq 28$ and $\simeq 48 \mu \mathrm{m}$, which is much smaller than the diameter of the liquid thread $(h \sim 1-10 \mathrm{~mm}$ ) while their length-to-diameter ratio, $a=l / d$, is varied between $\simeq 10$ and $\simeq 100$, see figure $1(d)$ and table I which also provides combinations of the relevant parameters. The fiber ends are slightly tapered because of the cutting process by which they are manufactured. They are suspended in a Newtonian liquid having a density matching their own density $\left(\rho=1140 \mathrm{~kg} / \mathrm{m}^{3}\right)$ and which wets them well. The liquid is an aqueous solution of PEGPG $(3.9 \mathrm{~kg} / \mathrm{mol}$ poly(ethylene glycol-ran-propylene glycol)-monobutylether by Sigma-Aldricht) or of Ucon oil 


$\begin{array}{cccccc} & d(\mu \mathrm{m}) & a=l / d & \phi / 100 & a \phi & a^{2} \phi \\ (\text { i }) & 28.9 \pm 1.2 & 10.8 \pm 1.8 & 1-24 & 0.108-2.59 & 1.16-27.9 \\ \text { (ii) } & 28.3 \pm 0.9 & 27.6 \pm 0.9 & 0.3-7 & 0.083-1.93 & 2.28-53.3 \\ \text { (iii) } & 28.1 \pm 1.3 & 106 \pm 5.5 & 0.08-2 & 0.085-2.12 & 8.96-224 \\ \text { (iv) } & 48.1 \pm 4.2 & 11.5 \pm 1.1 & 5 & 0.57 & 6.61\end{array}$

Table I. Characteristics of the fibers (the \pm symbol indicates the standard deviation over 30 fibers) and concentration ranges of the suspensions.

(polyalkylene glycol-composed lubricant Ucon 75-H 90000 by Dow) with $\mathrm{ZnCl}_{2}$ (Zinc-Chloride, Sigma-Aldricht). Its viscosity, $\eta_{0}$, is varied between 0.13 and $3.1 \mathrm{~Pa}$ s in order to obtain an effective viscosity of the suspension in the range where the measurements on the jet are accurate (see below) and those on the capillary bridge are free of inertial effects (see below). The particle volume fraction, $\phi$, is varied between $\sim 10^{-3}-10^{-2}$ and a value close to the largest volume fraction at which the suspension could be processed without noticeable aeration or self-filtration, i.e. 0.24, 0.07 , and 0.02 for $l / d=11,28$, and 106, respectively. The suspensions are prepared at the desired volume fraction and poured in a plastic syringe which is connected to the jet or bridge setups. Note that for the highest volume fractions $(\gtrsim 0.2)$, clear non-uniformities in the dispersion of the fibers are observed. Apparent clusters of fibers, with a typical dimension of a few fiber lengths, are found to form and to vanish dynamically as the suspension is gently mixed.

The jet setup is sketched in figure 1(a). It consists in forming a quasi-steady jet by extruding a fiber suspension through a vertical nozzle with a constant flow rate and observing the gravitational thinning of the jet. The suspension is extruded through a cylindrical nozzle having an inner diameter $h_{0}=11.6 \mathrm{~mm}$ (and a length $\simeq 30 \mathrm{~mm}$ ). A constant flow rate with mean velocity at the nozzle $u_{0} \sim 1 \mathrm{~cm} / \mathrm{s}$ is imposed with the help of a rigid piston and a high-precision translation stage. The thinning of the jet is imaged from a side view using a camera, see figure $2(a)$, and the thickness profile $h(z)$ is obtained by detecting the jet contour. The extensional effective viscosity is obtained with the same procedure as in [8] (also used previously by [17]), by comparing the stretching of the suspension jet with that expected for a Newtonian liquid. The quasi-steady velocity profile of the jet, $u(z)$, is obtained from the measurement of the thickness profile $h(z)$, by making use of the constant flow rate assumption, $u(z)=u_{0} h_{0}^{2} / h(z)^{2}$, and averaging over a sufficient time $\left(\sim 10 \mathrm{~s} \gg h_{0} / u_{0}\right)$. It has been verified that very close values are obtained by measuring directly the velocity from the displacement of the surface corrugations of the jet [8]. The reference Newtonian profile is obtained from the unidimensional, slender-slope approximation of the momentum and mass equations [22],

$$
u u_{z}=g+\frac{3 \eta_{\mathrm{e}}}{\rho} \frac{\left(h^{2} u_{z}\right)_{z}}{h^{2}}, \quad h^{2}=\frac{h_{0}^{2} u_{0}}{u},
$$

which is relevant in the present case of an elongated geometry $\left(h_{z} \ll 1\right)$ and negligible surface tension (the Weber number $\rho h_{0} U^{2} / \sigma$ is of order 20). Introducing the visco-gravity velocity scale, $U=\left(3 \eta_{\mathrm{e}} g / \rho\right)^{1 / 3}$, which determines whether the fall of the jet is limited by viscous stretching $(u \ll U)$ or is free $(u \gg U)$, and the visco-gravity length scale, $Z=\left(9 \eta_{\mathrm{e}}^{2} / \rho^{2} g\right)^{1 / 3}$, which represents the typical distance from the nozzle at which $U$ is reached for a slow extrusion $\left(u_{0} \ll U\right)$, these equations can be combined into [4]

$$
\left(\frac{u}{U}\right)^{\prime}=\frac{U}{u}+\left(\frac{(u / U)^{\prime}}{u / U}\right)^{\prime}
$$

where' denotes $Z \partial_{z}$. For a sufficiently long jet $\left(z \gg Z\right.$ ), equation (4) (with $u(0)=0$ and $\left.u^{\prime}(\infty) / U=0\right)$ determines a single universal velocity profile $u(z)$, whose scales, $U$ and $Z$, embed the extensional effective viscosity of the jet, $\eta_{\mathrm{e}}=\rho U^{3} / 3 g=\rho \sqrt{g Z^{3}} / 3$. The value of the latter is obtained from the best fit of the experimental profile with the solution of equation (4). In order to obtain accurate measurements the experiments are performed in the case $u_{0} \ll U$ for which most of the viscosity-limited stretching is observed and care is taken to verify the slenderness criterion $\left(Z \gg h_{0}\right)$.

In addition to the extensional measurements, the shear effective viscosity of the suspensions is measured with a shear cell sketched in figure 1(b) which enables large values of the shear gap and is calibrated with Newtonian liquids (see details in $[8,15]$ ). The shear viscosity is found to be almost independent of the gap (less than $5 \%$ variation when the gap size is increased from 2.5 to $5 \mathrm{~mm}$ ) and a slight shear thinning is observed for the largest solid fractions (typically, a 30\% decrease over one decade of shear rate for $\phi=0.20$ with $a=11$ ). Therefore, we report the average as well as the extreme values measured over the shear rate decade $1-10 \mathrm{~s}^{-1}$ which corresponds to the typical deformation rate $u_{z}$ in the jet.

The breakup dynamics is studied with the capillary bridge apparatus sketched in figure $1(c)$. The setup is identical to that used in [7]. It consists in letting a static pending suspension drop with a controlled size to merge with a bath 

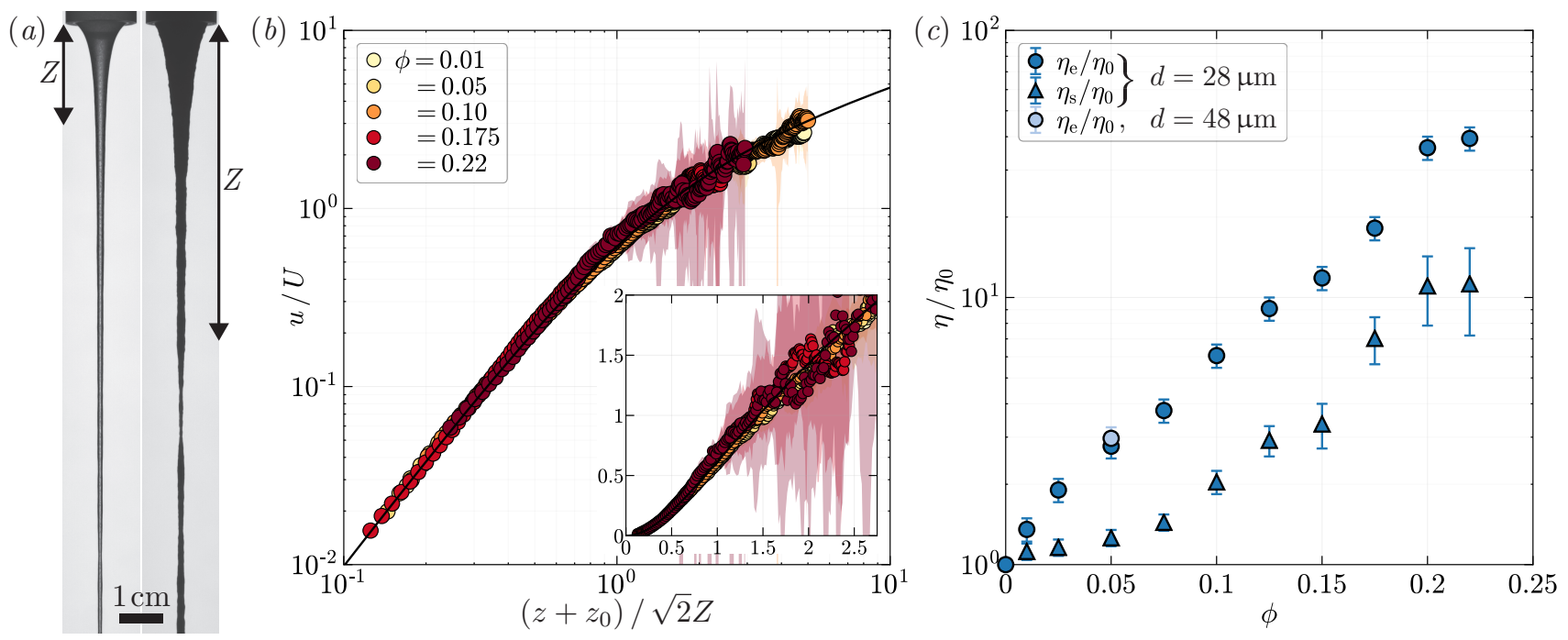

Figure 2. (a) Typical jets observed for $a \approx 11$ and $d \approx 28 \mu \mathrm{m}, \phi=0.01$ (left, $\eta_{0}=3.1 \mathrm{Pas}$ ) and $\phi=0.20$ (right, $\eta_{0}=0.66 \mathrm{Pas}$ ), and $u_{0}=1.36 \mathrm{~cm} / \mathrm{s}$. (b) Mean velocity versus distance to the nozzle for different volume fractions (same fibers as in (a), $\eta_{0}=3.1 \mathrm{Pas}$ for $\phi<0.10$ and $\eta_{0}=0.66 \mathrm{Pas}$ for $\left.\phi \geq 0.10\right)$. The solid line shows the velocity for a Newtonian liquid, equation (4), and $z_{0}$ determines the velocity at the nozzle $u_{0}$, i.e. $u\left(z_{0}\right)=u_{0}$. The shaded envelops indicate the standard deviation of $u_{0} h_{0}^{2} / U h^{2}$. Inset: Same data in linear scales. (c) Relative effective viscosity of the suspensions as measured from the jet velocity profile $\left(\eta_{\mathrm{e}} / \eta_{\mathrm{0}}\right)$ and with the shear cell $\left(\eta_{\mathrm{s}} / \eta_{0}\right)$.

of the same suspension and monitoring the thinning and pinching of the unstable bridge that is transiently formed by the coalescence. The drop is quasi-statically extruded from a stainless steel cylindrical nozzle with an outer diameter of $4.39 \mathrm{~mm}$ until its bottom lays $3.88 \mathrm{~mm}$ below the nozzle outlet. The suspension bath is then slowly raised (at typically $1 \mu \mathrm{m} / \mathrm{s}$ ) until the merging is triggered. The pinching dynamics of the bridge is imaged from a side view with a camera. The contour of the bridge is extracted and the time evolution of the minimal diameter of the bridge, $h_{\text {min }}$, is followed from $h_{\min }=h_{\text {ref }} \equiv 2.5 \mathrm{~mm}$ to the instant of the breakup, when $h_{\min }$ vanishes. The experiments are conducted with the smallest fibers $(a=10.8 \pm 1.8, d=28.9 \pm 1.2 \mu \mathrm{m})$ and for volume fractions, $\phi$, ranging from 0.015 to 0.24 . The suspending liquid viscosity $\left(\eta_{0}=0.13\right.$ or $3.05 \mathrm{Pas}$, see table III discussed in $\S$ III $)$ is chosen so as to obtain sufficiently large Ohnesorge numbers $\left(\eta_{\mathrm{e}} / \sqrt{\rho h_{\mathrm{ref}} \sigma} \gtrsim 10\right.$, where $\sigma \simeq 38$ and $42 \mathrm{mPa} / \mathrm{m}$, respectively, is the surface tension of the suspending liquid), which ensures that inertia does not affect the pinching. The typical deformation rates, $\dot{h}_{\min } / h_{\min } \sim 10^{-1}-1 \mathrm{~s}^{-1}$, are slightly smaller than those in the jet $\left(u_{z} \sim 1-10 \mathrm{~s}^{-1}\right)$. The viscous Newtonian reference dynamics is calibrated with the most viscous suspending liquid in the absence of particles. This ensures that the calibration is obtained for a surface tension that is within $10 \%$ that of the suspensions and a density that is equal.

For this range of parameters the Sperm number, $\left(128 \eta_{0} \dot{\epsilon} / E\right) a^{4} / \ln 2 a$, which determines whether the magnitude of the viscous stresses is sufficient to bend an isolated fiber [24] (with $\dot{\epsilon}=u_{z}$ or $\dot{h}_{\min } / h_{\min }$ the typical deformation rate and $E \approx 3-5 \mathrm{GPa}$ the Young modulus of the fibers), varies from $\sim 10^{-3}$ to $\sim 10$ (for $a$ ranging from 11 to 103). This is below the critical value for buckling $\approx 150[24]$. It should be stressed that this criterion is relevant only for isolated fibers that are aligned with a compressive direction of the flow, i. e. that are close to perpendicular to the thread axis.

\section{RESULTS}

\section{A. Jets}

Figure 2(a) shows the typical jet profiles observed for a suspension of fibers with $a=10.8 \pm 1.8$ and $d=28.9 \pm 1.2 \mu \mathrm{m}$ for both a dilute (left, $\phi=0.01$ ) and a concentrated (right, $\phi=0.20$ ) situation. In both cases, below a certain distance from the nozzle at which unsteady diameter modulations akin to a Plateau-Rayleigh instability eventually develop, an extended portion is observed where the jet is quasi-steady. Over this portion, which is a few nozzle diameters long, the jet stretches and thins monotonically with increasing distance from the nozzle and the quasi-steady thickness profile can be extracted. Figure 2(b) presents the stretching of the jet measured for the same fibers $(a=10.8 \pm 1.8$, $d=28.9 \pm 1.2 \mu \mathrm{m}$ ) and a few representative values of $\phi$ between 0.01 and 0.22 . All the velocity profiles (obtained using $u=u_{0} h_{0}^{2} / h^{2}$, see $\left.\S \mathrm{II}\right)$ are found to show the quadratic law $u / U \propto(z / Z)^{2}$ of the viscosity-limited stretching 


\begin{tabular}{|c|c|c|c|c|c|}
\hline$a=l / d$ & $a_{\mathrm{eff}}=\sqrt{\frac{\left\langle l^{3}\right\rangle}{\left\langle l d^{2}\right\rangle}}$ & $d(\mu \mathrm{m})$ & $\phi / 100$ & $\eta_{0}(\mathrm{Pas})$ & $\eta_{\mathrm{e}} / \eta_{0}$ \\
\hline 10.8 & 11.2 & 28.9 & 1 & 3.10 & 1.35 \\
\hline 11 & " & 11 & 2.5 & 3.10 & 1.90 \\
\hline "1 & "1 & " & 5 & 3.10 & 2.77 \\
\hline " & "1 & " & 7.5 & 3.10 & 3.77 \\
\hline " & " & "1 & 10 & 0.66 & 6.1 \\
\hline " & " & " & 12.5 & 0.66 & 9.1 \\
\hline " & "I & "1 & 15 & 0.60 & 11.8 \\
\hline "1 & " & "1 & 17.5 & 0.66 & 18.2 \\
\hline " & " & " & 20 & 0.66 & 36 \\
\hline "1 & "1 & "1 & 22 & 0.66 & 39 \\
\hline 27.6 & 27.7 & 28.3 & 0.3 & 3.10 & 1.61 \\
\hline "I & $" \prime$ & 11 & 1.3 & 3.10 & 3.16 \\
\hline "I & 'I & " & 7 & 0.62 & 16.9 \\
\hline 106 & 106 & 28.1 & 0.08 & 3.10 & 2.90 \\
\hline 'I & 11 & "I & 0.4 & 3.10 & 6.13 \\
\hline 'I & 'I & "I & 2 & 0.62 & 35.5 \\
\hline 11.5 & 11.4 & 48.1 & 5 & 3.10 & 2.97 \\
\hline
\end{tabular}

Table II. Extensional effective viscosities obtained with the jet setup.

regime $(u \ll U)$ and to be well fitted by equation (4) (solid black line) over a significant range of strain, $h_{0}^{2} / h^{2} \gg 10$. This suggests that the suspensions behave as an effective Newtonian liquid and that an extensional effective viscosity, $\eta_{\mathrm{e}}$, can be measured.

The value of $\eta_{\mathrm{e}}$ relative to the suspending liquid viscosity, $\eta_{0}$, is plotted as a function of the particle volume fraction in figure 2(c). It is found to increase strongly and continuously with increasing $\phi$, reaching typically 40 at $\phi=0.22$. In order to appreciate the influence of the particle size, figure $2(c)$ also reports the extensional viscosity measured for $\phi=0.05$ for particles that have a similar shape $(a \approx 11)$ but are almost twice as large $(d=48.1 \pm 4.2 \mu \mathrm{m}$ instead of $28.9 \pm 1.2 \mu \mathrm{m})$. Within the experimental uncertainty, the same value of $\eta_{\mathrm{e}} / \eta_{0}$ is obtained $(2.97 \mathrm{for} d=48.1 \pm 4.2$, vs 2.77 for $28.9 \pm 1.2 \mu \mathrm{m}$ ), which suggests that our rheological measurements are not influenced by finite size effects and probe the bulk rheology of the suspensions.

The extensional viscosity of the suspension obtained with the jet experiment, $\eta_{\mathrm{e}}$, can be compared to the shear effective viscosity, $\eta_{\mathrm{s}}$, measured with the shear cell. As shown in figure $2(c), \eta_{\mathrm{e}}$ remains larger than $\eta_{\mathrm{s}}$ over the whole range of $\phi$. More specifically, the increase in $\eta_{\mathrm{e}}$ is found to be much larger than in $\eta_{\mathrm{s}}$ for the smallest volume fractions $(\phi \lesssim 0.08)$, whereas for higher values of $\phi$ the two viscosities remain almost in the same ratio of approximately 4 . This suggests that, as expected in the dilute limit $[6,11]$, the fibers are more aligned with the straining direction in the jet than in the shear flow.

The dependence of the extensional effective viscosity on the fiber aspect ratio, $a$, is studied by increasing the length of the fibers while keeping their diameter $(d \approx 28 \mu \mathrm{m}$, see table I) and the range of $a \phi(0.1 \lesssim a \phi \lesssim 2$, see table I) almost unchanged. These data are provided in table II as a function of both $a$ and $\phi$. Figure $3(a)$ compares these measurements with the prediction for the dilute regime, by representing the specific effective viscosity, $\eta_{\mathrm{e}} / \eta_{0}-1$, as a function of the diluteness parameter, $\varepsilon Q(\varepsilon) a^{2} \phi$. For the smallest aspect ratio already discussed above $(a=10.8 \pm 1.8)$, $\eta_{\mathrm{e}} / \eta_{0}-1$ is found to increase close to linearly with $\phi$ up to $\phi \approx 0.08$. This suggests that particle interactions have a negligible influence over this range of $\phi$. This is also the case for the more slender fibers $(a=27.6 \pm 0.9$ and 106 \pm 5.5$)$, albeit over the whole range of volume fraction investigated. In the $\eta_{\mathrm{e}} / \eta_{0}-1$ vs $\varepsilon Q(\varepsilon) a^{2} \phi$ representation, all these measurements collapse onto the same line. They also collapse with the data previously obtained experimentally (by Weinberger [23], Mewis \& Metzner [14] and Pittman \& Bayram [17]) and from numerical simulation (by Mackaplow \& Shaqfeh [12]) for suspensions of thin and elongated fibers at lower volume fractions $(d \lesssim 10 \mu \mathrm{m}, a \sim 50-1200$ and $\phi \sim 5.10^{-4}-0.01$ ). All these measurements are found to agree, in trend and within a factor 2 , with the dilute regime prediction of Batchelor, equation (1), assuming that the fibers do not interact and are aligned with the jet axis [25].

By contrast, the values measured at high volume fractions $(\phi \gtrsim 0.08)$ for $a=10.8 \pm 1.8$ are found to deviate from the dilute regime prediction and to increase much more strongly than linearly with $\phi$. This deviation occurs for values of $\varepsilon Q(\varepsilon) a^{2} \phi$ typically above 10 , for which the viscosity for the more slender fibers is still following the dilute regime prediction. This indicates that the nonlinearity with volume fraction is not controlled by $a^{2} \phi$ only (or even less by $a \phi$ ). The departure from linearity is more evidenced in figure $3(b)$ by plotting the specific effective viscosity normalized by $\varepsilon Q(\varepsilon) a^{2}$ as a function of $\phi$. The semi-dilute models assuming sole hydrodynamics interactions, equations (2a-2b), are 

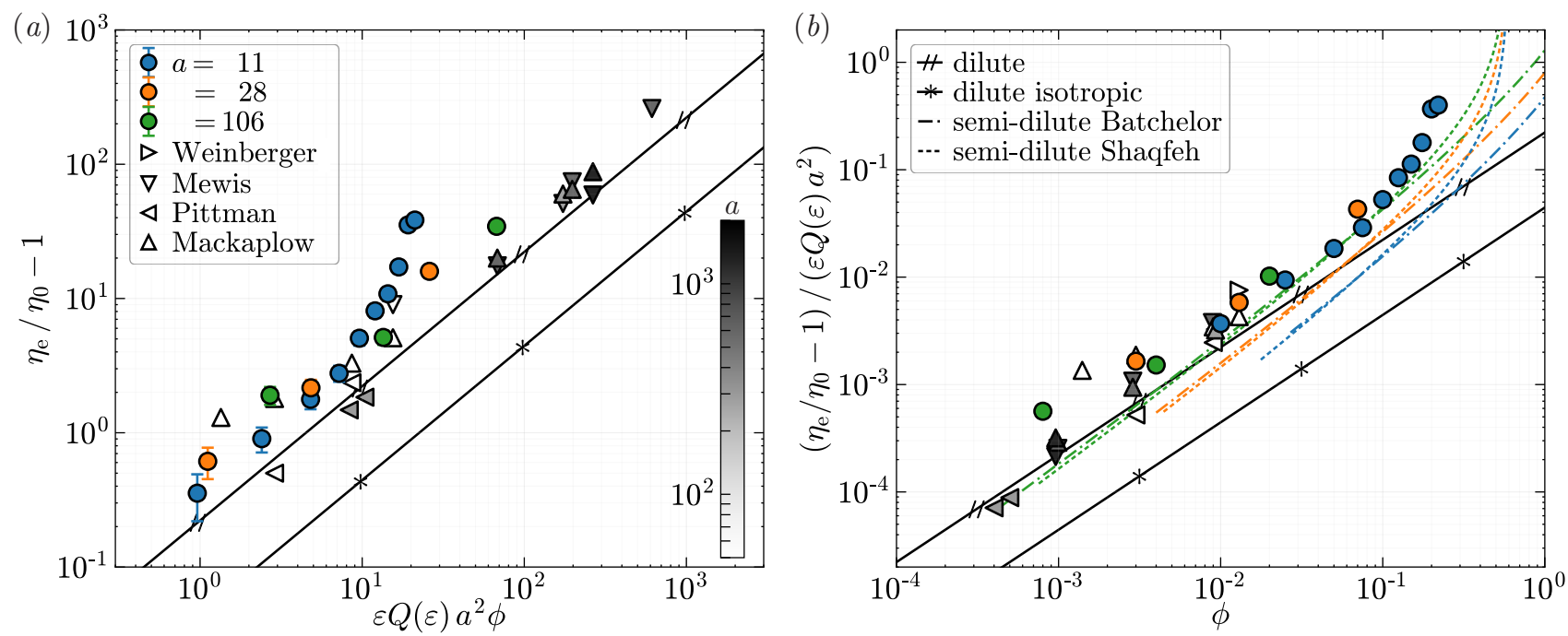

Figure 3. (a) Specific extensional effective viscosity as a function of the diluteness parameter, $\varepsilon Q(\varepsilon) a^{2} \phi$. The circles represent the values obtained with the jet setup for different aspect ratios but a similar particle diameter $(d \approx 28 \mu \mathrm{m})$. The triangles represent the experimental data by Weinberger [23], Mewis \& Metzner [14], Pittman \& Bayram [17], and the numerical data by Mackaplow \& Shaqfeh [12] (the value of $a$ is given by the symbol darkness as indicated in the scale bar). The solid lines show the predictions for the dilute regime with aligned, equation (1), and isotropic fibers (see text in $\S \mathrm{I}$ ). (b) Same viscosity as in $(a)$ normalized by $\varepsilon Q(\varepsilon) a^{2}$ as a function of $\phi$. The dashed-dotted and doted lines are the predictions for the semi-dilute regime, equations $(2 \mathrm{a}-2 \mathrm{~b})$, at the same aspect ratios as in the experiments.

predicting a much weaker deviation from linearity than that observed in the experiment and thus both fail to capture the experimental behavior at large $\phi$.

\section{B. Capillary breakup}

We now turn to the capillary bridge experiments, with the aim of comparing the dynamics of pinching with that of jet stretching. Figure 4(b-c) shows the typical time-evolution of the capillary thinning observed for a suspension with $\phi=0.05$ and 0.24 , respectively, using the smallest fibers $(a=10.8 \pm 1.8, d=28.9 \pm 1.2 \mu \mathrm{m})$. As a reference, the dynamics in the absence of fibers $(\phi=0)$ is also presented in the pannel $(a)$. In the three cases, the bridge is shown for the same values of its minimal diameter $\left(h_{\min }=2.5,1,0.5\right.$ and $\left.0.1 \mathrm{~mm}\right)$ and the corresponding times to breakup are indicated in the images (note that the suspending liquid is $\approx 23$ times less viscous for $\phi=0.24$ than for $\phi=0$ and 0.05 ). For $\phi=0.05$, the bridge shape is found to remain similar to that of the Newtonian case until the neck has decreased to a few fiber widths. By contrast, for $\phi=0.24$, the bridge is found to be distorted at all times, showing a non-axisymmetric shape with irregular protrusion, even when $h_{\min } \gg l(\approx 290 \mu \mathrm{m})$. As illustrated in figures $4(d)$, this irregularity in the shape at high volume fraction $(\phi=0.24)$ is not deterministic. The bridge shape observed for the same value of $h_{\min }(=0.5 \mathrm{~mm})$ for four realizations of the experiment under the same conditions is found to differ strongly between each realization. This large variability in the shape results in a large variability in the temporal dynamics. Figure $4(e)$ compares the time evolution of $h_{\min }$ from the same reference diameter, $h_{\text {min }}=h_{\text {ref }}=2.5 \mathrm{~mm}$, to the pinch-off instant $\left(h_{\min }=0\right)$ for 16 breakup events. Both the whole duration of the breakup, which varies over a factor close to 3 , and the shape of the thinning curve, show a large variability around the average dynamics indicated by the large symbols.

The influence of the particle volume fraction is summarized in figure $4(f)$ which compares the thinning dynamics obtained for $\phi$ ranging from 0 (Newtonian reference case) to 0.24 . In the inset the time is nondimensionalized with the averaged initial thinning rate, $\left.\dot{h}_{\min }\right|_{h_{\min }=h_{\text {ref }}}$, estimated around $h_{\min }=h_{\text {ref }}$. This scale emphasizes the influence of the particle volume fraction on the variability of the dynamics. Relative to the mean time (symbols), the standard deviation of $t$ at a given value of $h_{\min }$ (shaded envelop) increases continuously with increasing $\phi$. The inset in figure $4(f)$ also evidences that the shape of the thinning law is $\phi$-dependent. The larger the volume fraction becomes the more the thinning rate increases with decreasing $h_{\min }$. This relative acceleration is qualitatively similar to the behavior observed for spherical particles $[3,7,8,10,13]$. However, its interpretation in the present case is much more delicate because for a suspension of fibers at high $\phi$ even the initial shape of the bridge (for $h_{\text {min }} \gtrsim h_{\text {ref }}$ ) is found to be influenced by the particles, hence preventing direct comparisons.

Besides these differences in the shape of the thinning law, the most conspicuous influence of the fibers is to slow 

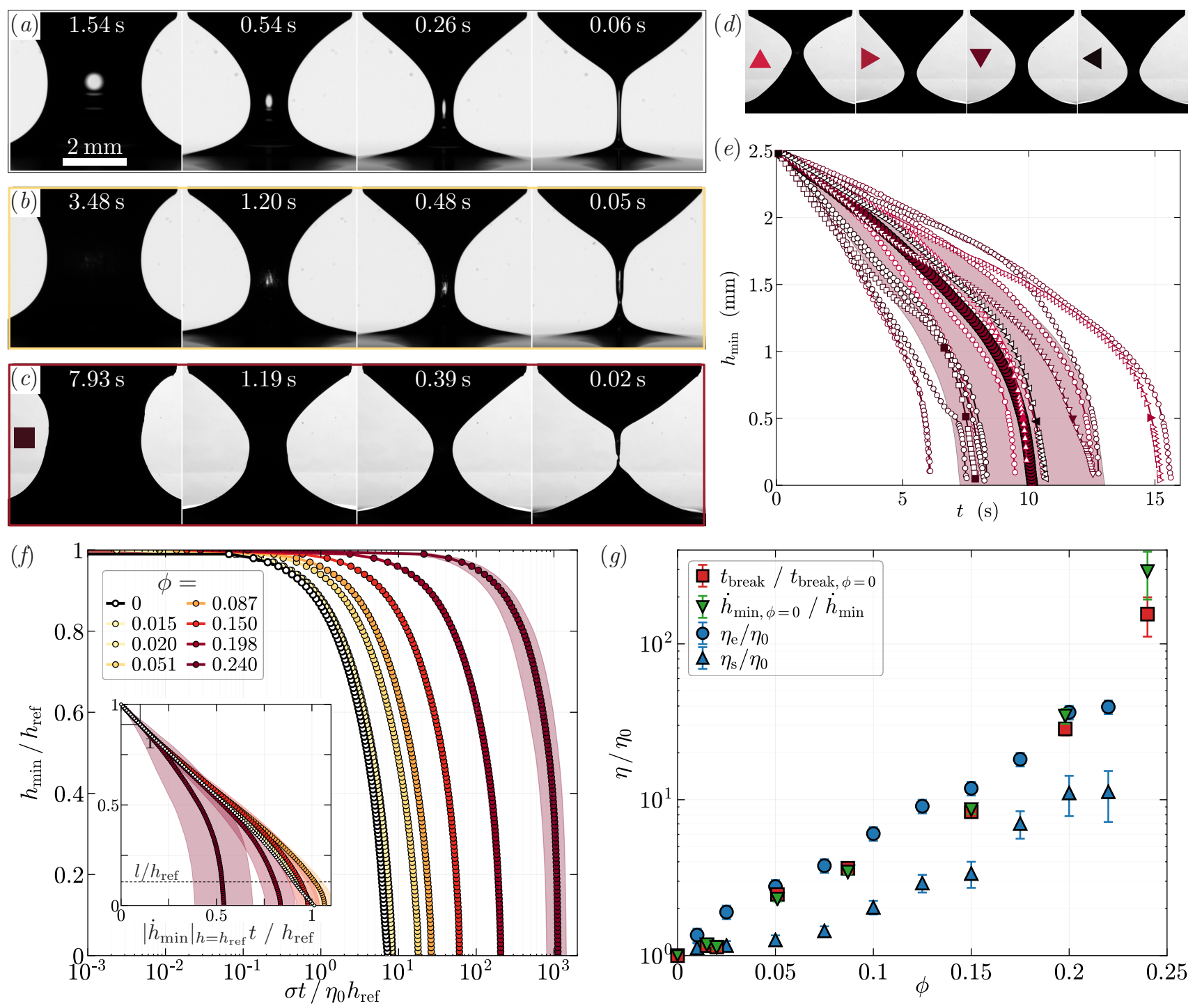

Figure 4. (a-c) Typical breakup dynamics of the capillary bridge. From top to bottom, $\phi=0,0.05$ and $0.24(a=10.8 \pm 1.8$, $d=28.9 \pm 1.2 \mu \mathrm{m}), \eta_{0}=3.05 \mathrm{Pas}$ for $(a-b)$ and $0.13 \mathrm{Pas}$ for $\left.(c)\right)$. The tags indicate the time, $t_{\mathrm{break}}-t$, remaining before break up (from left to right, $h_{\min }=2.5,1,0.5$ and $0.1 \mathrm{~mm}$, respectively). $(d)$ Variability in the bridge shape for four different breakup events with $\phi=0.24\left(h_{\mathrm{min}} \approx 0.5 \mathrm{~mm}\right)$. (e) Time evolution of the minimal diameter for 16 repetitions of the experiment with $\phi=0.24$ (the time origin is taken when $h_{\mathrm{min}}=h_{\mathrm{ref}}=2.5 \mathrm{~mm}$ ). The large filled symbols and the envelop show, respectively, the average and the standard deviation of $t$ at a given value of $h_{\min }$. The small filled symbols correspond to the images shown in $(c-d)$. $(f)$ Time evolution of the bridge minimal diameter for different particle volume fractions (average and standard deviation over 16 repetitions of the breakup, same value of $a$ and $d$ as in $(a-d))$. Inset: same data as a function of the time made dimensionless with the initial thinning rate $\left|\dot{h}_{\min }\right|_{h_{\min }=h_{\text {ref }}} / h_{\text {ref }}$ (the rate is measured between $h_{\text {min }}=h_{\text {ref }}$ and $\left.h_{\text {min }}=0.9 h_{\text {ref }}\right) .(g)$ Relative thinning rate as inferred from the mean breakup time $t_{\text {break }}$ and from the mean initial thinning rate $\left|\dot{h}_{\min }\right|_{h_{\min }=h_{\text {ref }}} / h_{\text {ref }}$ (same data for $\eta_{\mathrm{e}} / \eta_{0}$ and $\eta_{\mathrm{s}} / \eta_{0}$ as in figure $2 a$ ).

down the pinching dynamics. As shown in the main graph of figure $4(f)$, where time is made dimensionless by using the typical pinching duration for $\phi=0, \eta_{0} h_{\text {ref }} / \sigma$, the whole pinching duration increases continuously with increasing $\phi$ and is found to be typically $10^{2}$ times longer at $\phi=0.24$ than at $\phi=0$. The magnitude of this slowing of the dynamics is compared to the relative viscosity determined with the jet experiments in figure $4 g$, where both the relative breakup time, $t_{\text {break }} / t_{\text {break }, \phi=0}$, and the inverse of the relative initial thinning rate, $\dot{h}_{\min , \phi=0} / \dot{h}_{\text {min }}$, are reported as a function of $\phi$. These data are also provided in table III. These ratios are found to reflect quantitatively the extensional effective viscosity of the suspension, up to the largest volume fraction at which the two experiments can be compared $(\phi=0.20)$. This indicates that, in spite of the non-uniformity of the flow and of the differences with a Newtonian effective dynamics that have been mentioned above, the timescale of the pinching is well captured 


\begin{tabular}{cccc}
$\phi / 100$ & $\eta_{0}(\mathrm{Pas})$ & $t_{\text {break }} / t_{\text {break }, \phi=0}$ & $\dot{h}_{\text {min }, \phi=0} / \dot{h}_{\text {min }}$ \\
\hline 1.5 & 3.05 & $1.16 \pm 0.03$ & $1.18 \pm 0.03$ \\
2 & 3.05 & $1.13 \pm 0.04$ & $1.13 \pm 0.04$ \\
5.1 & 3.05 & $2.47 \pm 0.14$ & $2.31 \pm 0.09$ \\
8.7 & 3.05 & $3.63 \pm 0.15$ & $3.44 \pm 0.10$ \\
15 & 3.05 & $8.4 \pm 0.5$ & $8.7 \pm 0.6$ \\
19.8 & 3.05 & $28.5 \pm 2.7$ & $34.5 \pm 3.8$ \\
24 & 0.13 & $155 \pm 44$ & $291 \pm 99$
\end{tabular}

Table III. Extensional effective viscosities obtained with the capillary bridge setup $(a=10.8 \pm 1.8, d=28.9 \pm 1.2 \mu \mathrm{m})$. The value for the breakup time and initial thinning rate are averaged over 16 measurements and the value after the \pm symbol indicates the standard deviation.

by the extensional bulk effective viscosity.

\section{CONCLUSIONS}

Our measurements of the extensional bulk effective viscosity for moderate aspect ratios of the fibers and relatively high volume fractions $\left(a \sim 10-10^{2}\right.$ and $\phi$ up to 0.24$)$ complement those already available in the literature for more dilute suspensions of more slender fibers $\left(a \sim 10^{2}-10^{3}, \phi \sim 10^{-4}-10^{-2},[14,17,23]\right)$. Except for the most concentrated suspensions $(\phi \gtrsim 0.08, a=\approx 11)$, all the measurements are close to the dilute regime prediction assuming non-interacting fibers aligned with the strain direction. By contrast, for those most concentrated suspensions the specific viscosity, $\eta_{\mathrm{e}} / \eta_{0}-1$, increases nonlinearly with $\phi$ and much more strongly than predicted by the semidilute models assuming purely hydrodynamic long distance interactions. This suggests that lubrication, contact interactions between the fibers, or intrinsic non-uniformities in the distribution of the fibers need to be considered in this concentrated regime.

Observation on the thinning of a capillary bridge (for $a \approx 11$ ) reveals a significant departure from the Newtonian effective dynamics at solid volume fractions $\sim 0.20$ that are significantly lower than the critical volume fraction reported in a pure shear flow, $\sim 0.45[21]$, or the random packing volume fraction reported for dry fibers, $\sim 0.40$ [19]. This departure manifests in less slender bridge shapes and in an acceleration of the thinning rate during the pinch-off $\left(\left|\ddot{h}_{\text {min }}\right|>0\right)$. For the most concentrated suspensions, the departure is observed even when the size of the flow is still much larger than the fiber length $\left(h_{\min } / l \gtrsim 10\right)$, and is characterized by an important random feature producing a large variability in the shape of the bridge and in its temporal thinning law. However, in spite of these variabilities and of the non-uniformity and significant tridimensionality of the flow in the bridge, the total duration of the pinching is found to be in good quantitative agreement with the duration expected from a Newtonian effective dynamics limited by the extensional viscosity, $\eta_{\mathrm{e}}$.

[1] G. Batchelor. Slender-body theory for particles of arbitrary cross-section in Stokes flow. J. Fluid Mech., 44:419-440, 1970.

[2] G. Batchelor. The stress generated in a non-dilute suspension. J. Fluid Mech., 46:813-829, 1971.

[3] C. Bonnoit, T. Bertrand, E. Clément, and A. Lindner. Accelerated drop detachment in granular suspensions. Phys. Fluids, 24:043304, 2012.

[4] D. Brown. A study of the behavior of a thin sheet of moving liquid. J. Fluid Mech., 10:297-305, 1961.

[5] J. Burgers. On the motion of small particles of elongated form suspended in a viscous liquid. Kon. Ned. Akad. Wet. Verhand.(Eerste Sectie), 16:113-184, 1938.

[6] J. Butler and B. Snook. Microstructural dynamics and rheology of suspensions of rigid fibers. Annu. Rev. Fluid Mech., 50:299-318, 2018.

[7] J. Château, É. Guazzelli, and H. Lhuissier. Pinch-off of a viscous suspension thread. J. Fluid Mech., 852:178-198, 2018.

[8] J. Château and H. Lhuissier. Breakup of a particulate suspension jet. Phys. Rev. Fluids, 4:012001, 2019.

[9] R. Cox. The motion of long slender bodies in a viscous fluid Part 1. General theory. J. Fluid Mech., 44:791-810, 1970.

[10] R. Furbank and J. Morris. An experimental study of particle effects on drop formation. Phys. Fluids, 16:1777, 2004.

[11] G. Jeffery. The motion of ellipsoidal particles immersed in a viscous fluid. Proc. R. Soc. A, 102:161-179, 1922.

[12] M. Mackaplow and E. Shaqfeh. A numerical study of the rheological properties of suspensions of rigid, non-Brownian fibres. J. Fluid Mech., 329:155-186, 1996.

[13] W. Mathues, C. McIlroy, O. G. Harlen, and C. Clasen. Capillary breakup of suspensions near pinch-off. Phys. Fluids, 27:093301, 2015.

[14] J. Mewis and A. Metzner. The rheological properties. J. Fluid Mech., 62:593-600, 1974. 
[15] S. Palma and H. Lhuissier. Dip-coating with a particulate suspension. J. Fluid Mech., 869:R3, 2019.

[16] C. Petrie. The rheology of fibre suspensions. J. Non-Newt. Fluid Mech., 87:369-402, 1999.

[17] J. Pittman and J. Bayram. Extensional Flow of Polydisperse Fiber Suspensions in Free-Falling Liquid Jets. Int. J. Multiph. Flow, 16:545-559, 1990.

[18] R. Powell. Rheology of suspensions of rodlike particles. Journal of Statistical Physics, 62:1073-1094, 1991.

[19] O. Rahli, L. Tadrist, and R. Blanc. Experimental analysis of the porosity of randomly packed rigid fibers. $C$. $R$. Acad. Sci. Paris, 327:725-729, 1999.

[20] E. Shaqfeh and G. Fredrickson. The hydrodynamic stress in a suspension of rods. Phys. Fluids A, 2:7-24, 1990.

[21] F. Tapia, S. Shaikh, J. E. Butler, O. Pouliquen, and É. Guazzelli. Rheology of concentrated suspensions of non-colloidal rigid fibres. J. Fluid Mech., 827:725, 2017.

[22] J. Trouton. On the coefficient of viscous traction and its relation to that of viscosity. Proc. R. Soc. A, 77:426-440, 1906.

[23] C. Weinberger and J. Goddard. Extensional flow behavior of polymer solutions and particle suspensions in a spinning motion. Int. J. Multiph. Flow, 1:465-486, 1974.

[24] Y. Young and M. Shelley. Stretch-coil transition and transport of fibers in cellular flows. Phys. Rev. Lett., 99:849, 2007.

[25] Note that the difference between the experimental values and the dilute regime prediction cannot be attributed to the dispersion in the fibers dimensions. As shown in table II, the effective value of the aspect ratio, $a_{\text {eff }}=\sqrt{\left\langle l^{3}\right\rangle /\left\langle l d^{2}\right\rangle}$ obtained by weighting each fiber proportionally to its contribution to the increase in viscosity in equation (1), is very close to the simple average of $a=l / d$ used for the comparisons in figure 3 . 